\title{
Enhanced Situational Awareness: Application of DDDAS Concepts to Emergency and Disaster Management
}

\author{
Gregory R. Madey ${ }^{1}$, Albert-László Barabási ${ }^{2}$, Nitesh V. Chawla ${ }^{1}$, \\ Marta Gonzalez ${ }^{2}$, David Hachen ${ }^{3}$, Brett Lantz ${ }^{3}$, Alec Pawling ${ }^{1}$, \\ Timothy Schoenharl ${ }^{1}$, Gábor Szabó ${ }^{2}, \mathrm{Pu} \mathrm{Wang}^{2}$, and Ping Yan ${ }^{1}$ \\ ${ }^{1}$ Department of Computer Science \& Engineering \\ University of Notre Dame, Notre Dame, IN 46556, USA \\ \{gmadey, nchawla, apawling, tschoenh, pyan\}@nd.edu \\ ${ }^{2}$ Department of Physics \\ University of Notre Dame, Notre Dame, IN. 46556, USA \\ \{alb, m.c.gonzalez, gabor.szabo, pwang2\}@nd.edu \\ ${ }^{3}$ Department of Sociology \\ University of Notre Dame, Notre Dame, IN 46556, USA \\ \{dhachen, blantz\} and.edu
}

\begin{abstract}
We describe a prototype emergency and disaster information system designed and implemented using DDDAS concepts. The system is designed to use real-time cell phone calling data from a geographical region, including calling activity - who calls whom, call duration, services in use, and cell phone location information - to provide enhanced situational awareness for managers in emergency operations centers (EOCs) during disaster events. Powered-on cell phones maintain contact with one or more within-range cell towers so as to receive incoming calls. Thus, location data about all phones in an area are available, either directly from GPS equipped phones, or by cell tower, cell sector, distance from tower and triangulation methods. This permits the cell phones of a geographical region to serve as an ad hoc mobile sensor net, measuring the movement and calling patterns of the population. A prototype system, WIPER, serves as a test bed to research open DDDAS design issues, including dynamic validation of simulations, algorithms to interpret high volume data streams, ensembles of simulations, runtime execution, middleware services, and experimentation frameworks [1].
\end{abstract}

\section{Introduction}

For disaster and emergency response managers to perform effectively during an event, some of their greatest needs include quality communication capabilities and high levels of situational awareness [2-4]. Reports from on-scene coordinators, first responders, public safety officials, the news media, and the affected population can

${ }^{*}$ The material presented in this paper is based in part upon work supported by the National Science Foundation, the DDDAS Program, under Grant No. CNS-050312. 
provide managers with point data about an emergency, but those on-scene reports are often inaccurate, conflicting and incomplete with gaps in geographical and temporal coverage. Additionally, those reports must be fused into a coherent picture of the entire affected area to enable emergency managers to effectively respond.

The prototype Wireless Phone Based Emergency Response System (WIPER) is designed using the concepts of Dynamic Data Driven Application Systems (DDDAS) with the goal of enhancing situational awareness of disaster and emergency response managers [5, 6]. The subsequent sections review open research issues with the DDDAS concept and design and development features of the WIPER prototype that address some of those open issues. Those features include an analysis of the local and the global structure of a large mobile communication network, several algorithms for detecting anomalies in streaming data, agent-based simulations for classification and prediction, a distributed system design using web services, and the design and implementation of the WIPER DSS interface.

\section{Dynamic Data Driven Application Systems}

Dynamic data driven application systems (DDDAS) were explored in detail in two NSF workshops: one in early 2000 [7] and one in 2006 [1]. The first workshop concluded that the DDDAS concept offered the potential of greatly improving the accuracy and efficiency of models and simulations. The workshop final report identified more research in the areas of 1) dynamic, data driven application technologies, 2) adaptive algorithms for injecting and steering real-time data into running simulations, and 3) systems software that supports applications in dynamic environments. At following conferences, initial research and applications exploring these research areas were reported [8]. A fourth area of research important to the DDDAS concept emerged, that of measurement systems; the dynamic steering of the data collection needed by the simulations may require improvements in measurement, observation and instrumentation methods. In 2004, Darema described the DDDAS concept as:

Dynamic Data Driven Application Systems (DDDAS) entails the ability to incorporate additional data into an executing application - these data can be archival or collected on-line; and in reverse, the ability of applications to dynamically steer the measurement process. The paradigm offers the promise of improving modeling methods, and augmenting the analysis and prediction capabilities of application simulations and the effectiveness of measurement systems. This presents the potential to transform the way science and engineering are done, and induce a major impact in the way many functions in our society are conducted, such as manufacturing, commerce, hazard management, and medicine [8].

The second major NSF workshop on DDDAS (2006) highlighted progress to date, and research yet to be conducted on open issues in the DDDAS concept $[1,9]$. The prototype WIPER system explores all four research areas relevant to the DDDAS concept $[5,6]$ and many of the open DDDAS research issues. Those open DDDAS research areas include: 1) Dynamic and continuous validation of models, algorithms, systems, and system of systems: WIPER uses an ensemble of agent-based simulations 
to test hypothesis about the emergency event, dynamically validating those simulations against streaming data, 2) Dynamic data driven, on demand scaling and resolution - the WIPER simulations request detailed data from the data source, providing higher resolution where needed to support dynamic validation, 3) Data format: collections taken across different instrument types can range in format and units - the WIPER data source includes multiple types of data, including location and movement, cell call data, service type, e.g., voice, data, SMS, 4) System Software, especially runtime execution, and middleware service and computational infrastructure WIPER is employing a novel application of web services (SOA), messaging, and asynchronous Javascript and XML (AJAX) to integrate heterogeneous services and user displays, 5) Mathematical and Statistical Algorithms, especially advances in computational simulation tools for modeling, and system and integration of related algorithms - WIPER includes new algorithms for monitoring streaming data [10,11] and new insight from mobile phone call data that will be incorporated into planned algorithms for anomaly detection $[12,13]$.

\section{Data}

The WIPER system uses both actual call and location data provided by a cellular carrier and synthetic data to simulate emergencies. The actual data is used to calibrate the generation of synthetic data and to help design the anomaly detection algorithms. An anomaly, a possible indication of an emergency, triggers the execution of an ensemble of simulations, each dynamically validated against new streaming data. All data is anonymized to protect privacy. During development, testing and evaluation,

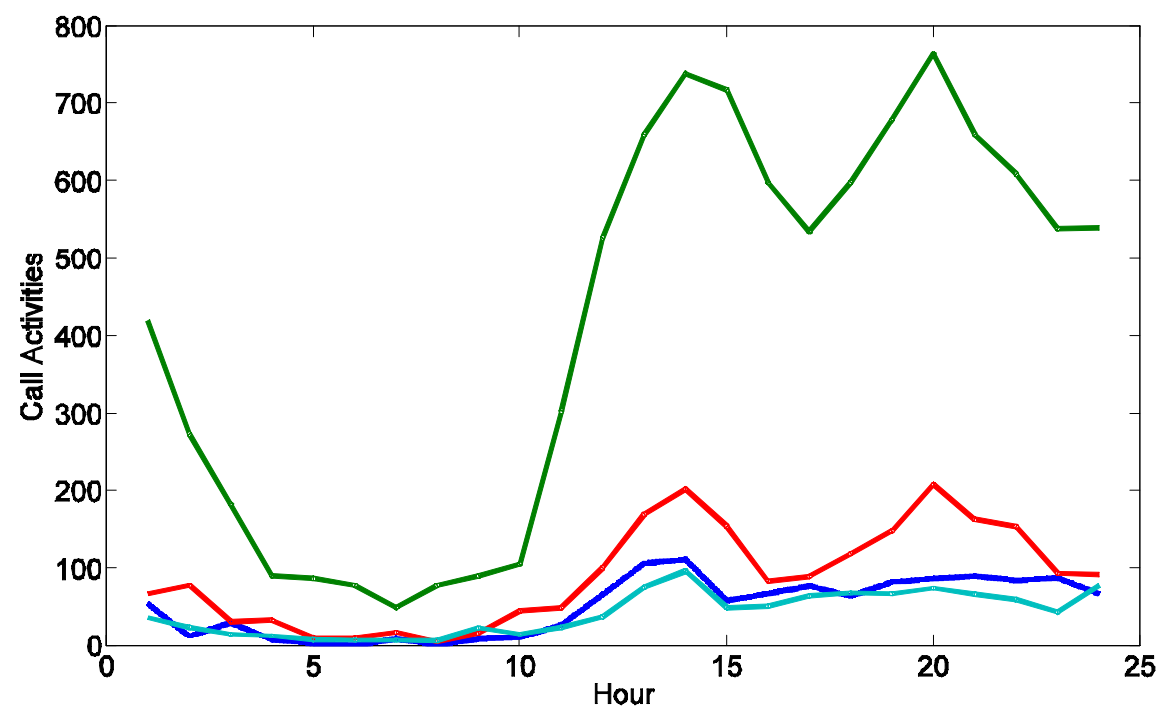

Fig. 1. Actual mobile phone calling activity per hour from a small city (20,000 population) for a 24 hour period starting at midnight. The four data series are associated with 4 different cell towers (all cell sectors combined for each tower). This data is used to calibrate synthetic data and to validate anomaly detection algorithms. 
the data is stored in a database with software modules streaming the data to simulate the real-time data streams the system would see if deployed. The data that the WIPER system uses is aggregate in nature, does not track individual cell phones by actual user ID, and does not include the content of phone calls or messages. An example of the call activity over a 24 hour period or a small city with 4 cell towers is shown in Fig. 1. The synthetic data is validated against such data.

\section{Design}

The design and operation of the WIPER DDDAS prototype is shown schematically in Fig. 2. The system has five components: 1) Data Source, 2) Historical Data Storage, 3) Anomaly Detection and Alert System (DAS), 4) Simulation and Prediction System (SPS), and 5) Decision Support (DSS) with Web Interface. Additional details about the operation of each component can be found elsewhere [5, 6]. The Simulation and Prediction System is triggered by the detection of an anomaly and an alert signal from the DAS. An ensemble of agent-based simulations evaluates possible explanations for the anomaly and alert. The dynamically validated simulation is used to classify the event and to predict its evolution. This and other WIPER provided information is displayed on a web-based console to be used by emergency managers for enhance situational awareness as shown in Fig. 3.

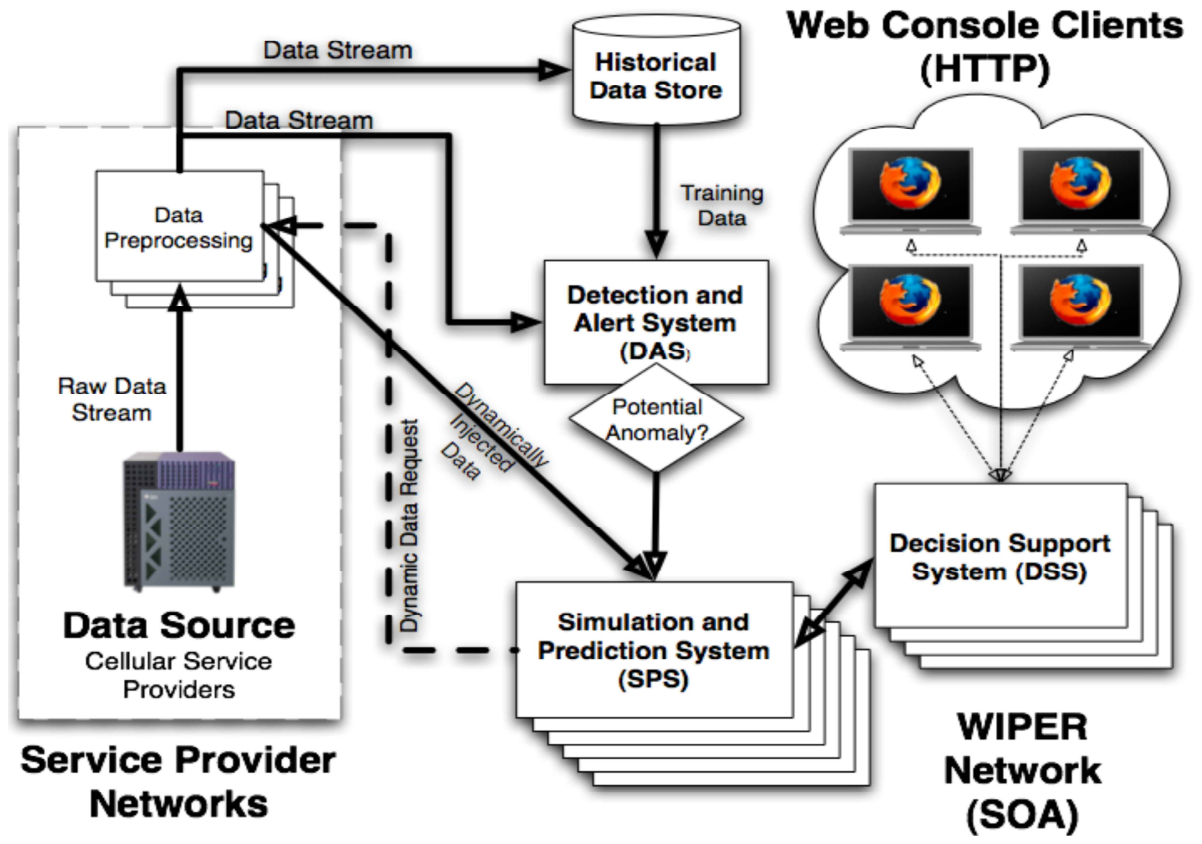

Fig. 2. Architecture of the WIPER DDDAS system: Either synthetic data or actual data from the cell phone providers is used for training, real time anomaly detection and dynamic validation of the simulation and prediction system 


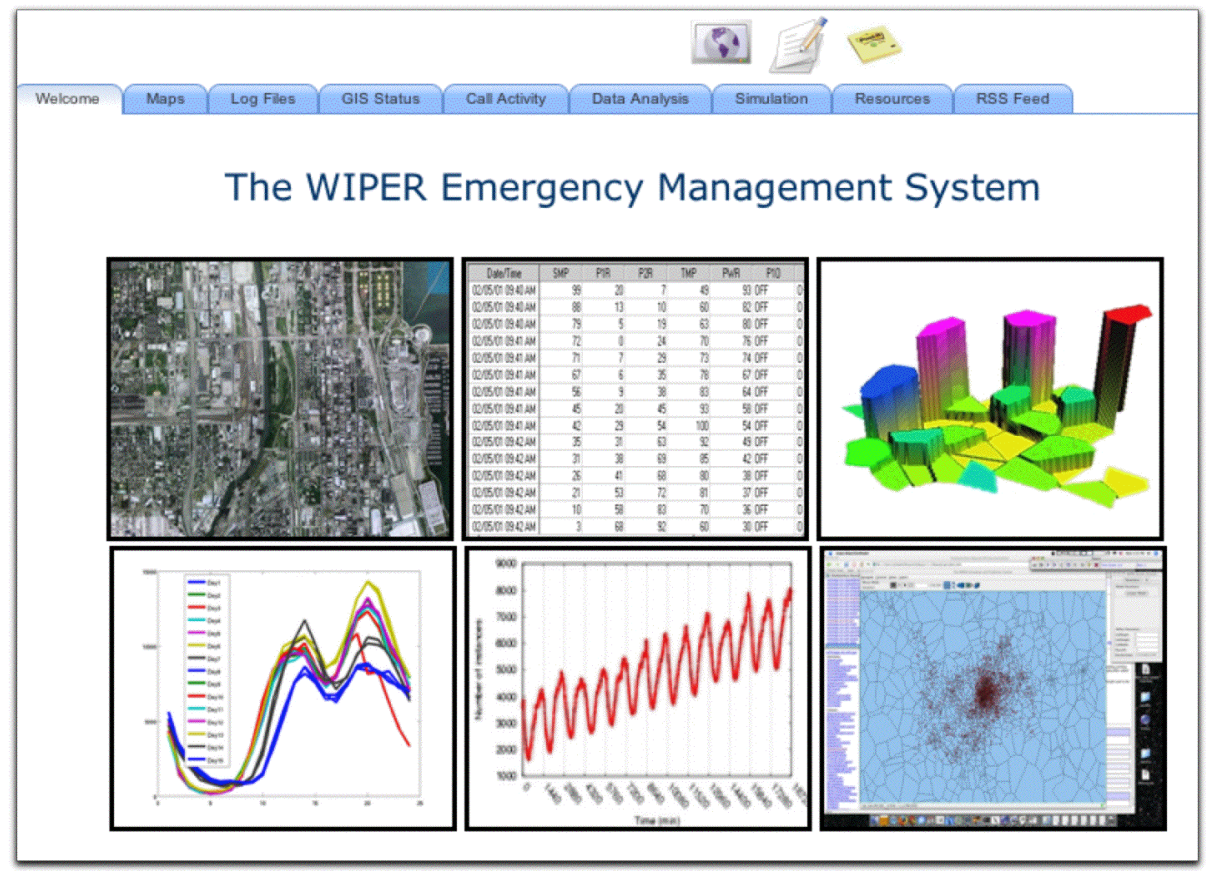

Fig. 3. The WIPER DSS console is web based, using asynchronous Javascript and XML (AJAX). Emergency managers can view streaming call activity statistics from the area of an incident, maps, weather information, GIS visualizations, and animations derived from agentbased simulation used to predict the evolution of an incident.

\section{Data, Algorithms and Analysis}

The DDDAS concept is characterized by dynamic data, the first two D's in DDDAS. In the WIPER system the dynamic data is cell phone activity: temporal calling patterns, statistics, locations, movement, and the evolving social networks of callers. In order to improve the detection and classification of anomalies and to dynamically validate predictive simulations, the WIPER project has conducted to date, five different, but complementary investigations. Each contributes understanding and methods for anomaly detection on streaming cell phone activity data.

\subsection{Structure and Tie Strengths in Mobile Communication Networks}

Cell phone calling activity databases provide detailed records of human communication patterns, offering novel avenues to map and explore the structure of social and communication networks potentially useful in anomaly detection. This investigation examined the communication patterns of several million of mobile phone users, allowing the simultaneous study of the local and the global structure of a society-wide communication network. We observed a coupling between interaction strengths and the network's local structure, with the counterintuitive consequence that social 
networks are robust to the removal of the strong ties, but fall apart following a phase transition if the weak ties are removed [13].

\subsection{Anomaly Detection Using a Markov Modulated Poisson Process}

Cell phone call activity records the behavior of individuals, which reflects underlying human activities across time. Therefore, they appear to have multi-level periodicity, such as weekly, daily, hourly, etc. Simple stochastic models that rely on aggregate statistics are not able to differentiate between normal daily variations and legitimate anomalous (and potentially crisis) events. In this investigation we developed a framework for unsupervised learning using Markov modulated Poisson process (MMPP) to model the data sequence and use the posterior distribution to calculate the probability of the existence of anomalies over time [11].

\subsection{Mapping and Visualization}

The cell phone calling data currently includes user locations and activity at a cellsized level of resolution. The size of a wireless cell can vary widely and depends on many factors, but these can be generalized in a simple way using a Voronoi diagram. A Voronoi lattice is a tiling of polygons in the plane constructed in the following manner: Given a set of points $\mathrm{P}$ (in our case, a set of towers) construct a polygon around each point in $\mathrm{P}$ such that for all points in the polygon around $\mathrm{p}_{0}$, the point is closer to $\mathrm{p}_{0}$ than to any other point in $\mathrm{P}$. Thus we can construct a tiling of a GIS space into cells around our towers. We build a 3D image based on the activity at the site of interest as shown in Fig. 4. This 3D view gives a good representation of the comparative activity levels in the cells $[6,14]$.

\subsection{Hybrid Clustering Algorithm for Outlier Detection on Streaming Data}

We developed a hybrid clustering algorithm that combines k-means clustering, the leader algorithm, and statistical process control. Our results indicate that the quality of the clusters produced by our algorithm are comparable to, or better than, those produced by the expectation maximization algorithm using sum squared error as an evaluation metric. We also compared the outlier set discovered by our algorithm with the outliers discovered using one nearest neighbor. While our clustering algorithm produced a number of significant false positive and false negatives, most of the outlier detected by our hybrid algorithm (with proper parameter settings) were in fact outliers. We believe that our approach has promise for clustering and outlier detection on streaming data in the WIPER Detection and Alert System [10].

\subsection{Quantitative Social Group Dynamics on a Large Scale}

Interactions between individuals result in complex community structures, capturing highly connected circles of friends, families, or professional cliques in social networks. Although most empirical studies have focused on snapshots of these communities, because of frequent changes in the activity and communication patterns of individuals, the associated social and communication networks are subject to constant evolution. Our knowledge of the mechanisms governing the underlying community 
dynamics is limited, but is essential for exploiting the real time streaming cell phone data for emergency management. We have developed a new algorithm based on a clique percolation technique, that allows, for the first time, to investigate in detail the time dependence of overlapping communities on a large scale and to uncover basic relationships of the statistical features of community evolution. The focus of this investigation on the networks formed by the calls between mobile phone users, observing that these communities are subject to a number of elementary evolutionary steps ranging from community formation to breakup and merging, representing new dimensions in their quantitative interpretation. We found that large groups persist longer if they are capable of dynamically altering their membership, suggesting that an ability to change the composition results in better adaptability and a longer lifetime for social groups. Remarkably, the behavior of small groups displays the opposite, the condition for stability being that their composition remains unchanged [12].

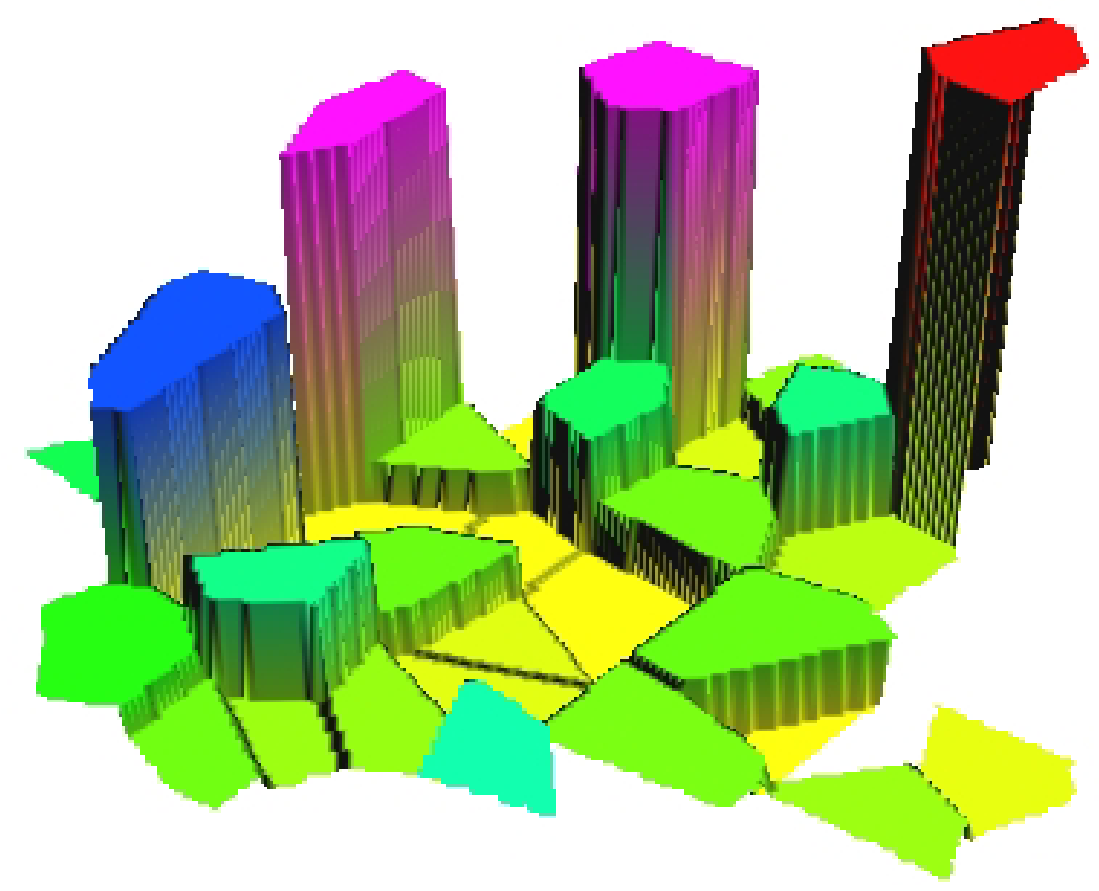

Fig. 4. A $3 \mathrm{D}$ view of activity in mobile phone cells. Each polygon represents the spatial area serviced by one tower. Cell color and height are proportional to the number of active cell phone users in that cell for a unit of time.

\section{Summary}

WIPER is designed for real-time monitoring of normal social and geographical communication and activity patterns of millions of cell phone users, recognizing unusual human agglomerations, potential emergencies and traffic jams. WIPER uses streams 
of high-resolution data in the physical vicinity of a communication or traffic anomaly, and dynamically injects them into agent-based simulation systems to classify and predict the unfolding of the emergency in real time. The agent-based simulation systems dynamically steer local data collection in the vicinity of the anomaly. Distributed data collection, monitoring, analysis, simulation and decision support modules are integrated to generate traffic forecasts and emergency alerts for engineering, public safety and emergency response personnel for improved situational awareness.

\section{References}

[1] NSF, "DDDAS Workshop Report," http://www.dddas.org/nsf-workshop2006/-wkshp_ report.pdf, 2006.

[2] J. Harrald and T. Jefferson, "Shared Situational Awareness in Emergency Management Mitigation and Response," in Proceedings of the 40th Annual Hawaii International Conference on Systems Sciences: Computer Society Press, 2007.

[3] Naval Aviation Schools Command, "Situational Awareness," http://wwwnt.cnet.navy. $\mathrm{mil} / \mathrm{crm} / \mathrm{crm} / \mathrm{stand} \_m a t / s e v e n \_s k i l l s / S A . a s p, 2007$.

[4] R. B. Dilmanghani, B. S. Manoj, and R. R. Rao, "Emergency Communication Challenges and Privacy," in Proceedings of the 3rd International ISCRAM Conference, B. Van de Walle and M. Turoff, Eds. Newark, NJ, 2006.

[5] G. Madey, G. Szábo, and A.-L. Barabási, "WIPER: The integrated wireless phone based emergency response system," Proceedings of the International Conference on Computational Science, Lecture Notes in Computer Science, vol. 3993, pp. 417-424, 2006.

[6] T. Schoenharl, R. Bravo, and G. Madey, "WIPER: Leveraging the Cell Phone Network for Emergency Response," International Journal of Intelligent Control and Systems, (forthcoming) 2007.

[7] NSF, "Workshop on Dynamic Data Driven Application Systems," www.cise.nsf.gov/ dddas, 2000.

[8] F. Darema, "Dynamic Data Driven Application Systems: A New Paradigm for Application Simulations and Measurements," in ICCS'04, Krakow, Poland, 2004.

[9] C. C. Douglas, "DDDAS: Virtual Proceedings," http://www.dddas.org/virtual_proceeings. html, 2006.

[10] A. Pawling, N. V. Chawla, and G. Madey, "Anomaly Detection in a Mobile Communication Network," Proceedings of the NAACSOS, 2006.

[11] Y. Ping, T. Schoenharl, A. Pawling, and G. Madey, "Anomaly detection in the WIPER system using a Markov modulated Poisson distribution," Working Paper, Notre Dame, IN: Computer Science \& Engineering, University of Notre Dame, 2007.

[12] G. Palla, A.-L. Barabási, and T. Viscsek, "Quantitative social group dynamics on a large scale," Nature (forthcoming), 2007.

[13] J.-P. Onnela, J. Saramäki, J. Hyvönen, G. Szabó, D. Lazer, K. Kaski, J. Kertész, and A.L. Barabási, "Structure and tie strengths in mobile communication networks," PNAS (forthcoming), 2007.

[14] T. Schoenharl, G. Madey, G. Szabó, and A.-L. Barabási, "WIPER: A Multi-Agent System for Emergency Response," in Proceedings of the 3rd International ISCRAM Conference, B. Van de Walle and M. Turoff, Eds. Newark, NJ, 2006. 